\title{
Kinetic roughening-like transition with finite nucleation barrier
}

\author{
James F. Lutsko, Vasileios Basios, and Grégoire Nicolis \\ Center for Nonlinear Phenomena and Complex Systems CP 231, \\ Université Libre de Bruxelles, Blvd. du Triomphe, 1050 Brussels, Belgium* \\ John J. Kozak \\ Dept. of Chemistry, DePaul University, \\ 243 South Wabash Avenue, Chicago, Illinois 60604, USA \\ Mike Sleutel and Dominique Maes \\ Flanders Interuniversity Institute for Biotechnology (VIB), \\ Vrije Universiteit Brussel, Pleinlaan 2 Building E, 1050 Brussel, Belgium
}

(Dated: November 21, 2018)

\begin{abstract}
Recent observations of the growth of protein crystals have identified two different growth regimes. At low supersaturation, the surface of the crystal is smooth and increasing in size due to the nucleation of steps at defects and the subsequent growth of the steps. At high supersaturation, nucleation occurs at many places simultaneously, the crystal surface becomes rough and the growth velocity increases more rapidly with increasing supersaturation than in the smooth regime. Kinetic roughening transitions are typically assumed to be due to the vanishing of the barrier for twodimension nucleation on the surface of the crystal. We show here, by means of both analytic mean field models and kinetic Monte Carlo simulations that a transition between different growth modes reminiscent of kinetic roughening can also arise as a kinetic effect occurring at finite nucleation barriers.
\end{abstract}

PACS numbers: 82.20.Wt, 81.10.Aj, 82.60.Nh

*Electronic address: jlutsko@ulb.ac.be URL: http://www.lutsko.com 


\section{INTRODUCTION}

Crystal growth takes place at the interface of the solid and liquid phases and is determined by the structure of the crystal surface, the temperature and the supersaturation. Each growth unit is incorporated into the crystal when the energy barrier for absorption is overcome by the unit's kinetic energy. For rough surfaces, such as K- and S-faces, the density of privileged sites, i.e. kinks, is high and growth is fast. In this work, however, we consider initially flat faces such as the F-faces. For these surfaces, at low supersaturation values, kink sites are scarce and the growth process is slow. Growth units encounter the kinks directly from solution, or via a random walk due to 2D-diffusion on the surface. New steps are either generated through spiral dislocations or 2D-nucleation[1 6] . The temperature is a determining factor. It is well known that there exists a critical value, $T_{R}$, where the step edge free energy vanishes and the crystal surface becomes rough at the growth unit scale. This is a thermodynamic phase transition, known as thermal roughening[7-12].

At constant temperature, the driving force is the chemical potential difference or supersaturation and an increase in the driving force will translate into a decrease in critical nucleus size. Since for temperatures less than $T_{R}$ the step edge's free energy is greater than zero, according to the Gibbs-Thomson relation [13, 14], an activation barrier for the formation of the critical two-dimensional nucleus exists and with it, a critical nucleus size larger than the crystal's individual building blocks. The size of this $2 \mathrm{D}$-critical nucleus is inversely proportional to the temperature and the supersaturation [1].

Recently, it has been reported for protein crystallization systems [15, 16] that for temperatures lower than $T_{R}$ there exists a critical supersaturation for which the size of the critical two-dimensional nucleus is reduced to the order of one growth unit and the activation barrier for two-dimensional nucleation essentially vanishes. Due to a large step density and a very small two-dimensional critical nucleus at elevated supersaturations, the surface becomes rough and offers many favorable sites almost uniformly distributed across the surface. Consequently, arriving molecules can be incorporated quasi at any site. This transition from a slow, layer-by-layer growth regime to a fast continuous growth regime at high driving forces is referred to as kinetic roughening transition [17-22].

Kinetic roughening for the case of crystallization from solution has been observed for many small molecules, i.e. $\mathrm{SiO}_{2}, \mathrm{Al}_{2} \mathrm{O}_{3}, \mathrm{ZnO}$ and $\mathrm{ZnS}$ [23, 24], n-paraffins [25, 26] and 
many others. For the case of macromolecules such as in protein crystallization, kinetic data of continuous growth has only been presented for lysozyme [15, 27, 28]. Very recently, quantitative data for both the classical layer-by-layer growth mechanism and the kinetic roughening regime for the crystallization of glucose isomerase from Streptomyces rubiginosus have been presented [16].

In this paper we show that kinetic roughening-like transitions in the presence of a $f$ nite nucleation barrier can occur owing to kinetic mechanisms still rendering possible the transition from slow layer-by-layer growth regime (low supersaturations) to a fast continuous growth regime (high supersaturations). The general scheme used views the process of crystal growth at constant temperature driven by the difference in chemical potential of the solution around the crystal surface and the reservoir of the surrounding liquid phase. The local environment around the surface incorporates the barriers, kinks and dislocations while relevant kinetic processes such as absorption, surface diffusion and nucleation contribute to the growth. A mean field model and a Monte Carlo simulation are proposed and serve as an illustrative generic explanation of the observed data from protein crystallization [16].

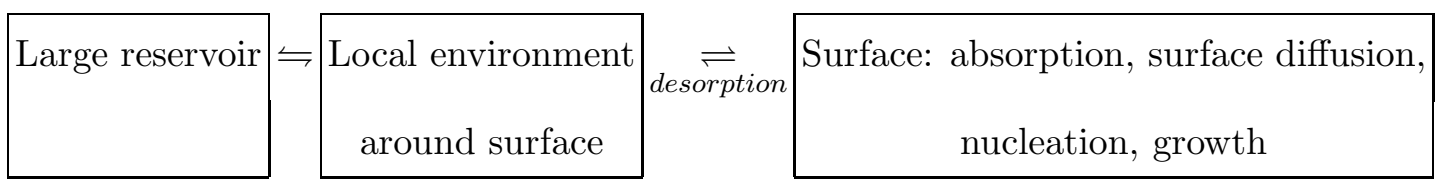

In Section II a minimal mean field model is developed incorporating cluster formation and possessing the two main asymptotic regimes, exhibiting the transition from low to high supersaturation growth. In Section III we investigate an extension of a "solid to solid" (SOS) Monte Carlo simulation linked to, but more general than, the mean field model, and accounting for kinetic roughening in the presence of finite nucleation barrier and dislocations. The main conclusions are summarized in Section IV.

\section{MEAN FIELD MODEL}

Our mean-field model of crystal growth is based on a generalization of the BurtonCabrera-Frank model for epitaxial growth[7, 29, 30] and is also motivated by theoretical work hinting at a competition between surface aggregation and the formation of cluster by direct incorporation of material from solution[31]. Let $X_{0}$ and $X$ denote, respectively, 
molecules in the bulk solution far from the growing crystal and in solution near the crystal surface; and $Y, C_{N}$ the concentration of molecules adsorbed onto the surface but not incorporated into the solid and of clusters constituting islands of solid material of size $N$. There are four basic physical processes that we consider. First is the diffusion of molecules between the bulk solution and the solution near the growing crystal, $X_{0} \rightleftarrows X$. Distinguishing between the bulk solution and that near the surface allows us to model the formation of a depletion layer as molecules are adsorped onto the surface and incorporated into the solid. The second process is that of adsorption from the solution onto the surface, $X \rightleftarrows Y$. We consider two pathways for the formation of an island of size $N$ : adsorption and aggregation, $X+(N-1) Y \rightleftarrows C_{N}$, and aggregation alone, $N Y \rightleftarrows C_{N}$. Denoting the concentrations of $X$ and $X_{0}$ by $x$ and $x_{0}$ respectively, the surface concentration of adsorbates and $N$-islands by $y$ and $c_{n}$, and assigning rate constants to each process gives the rate equations

$$
\begin{aligned}
\frac{d x}{d t} & =a x_{0}+k_{0}^{\prime} y-\left(a^{\prime}+k_{0}\right) x+k_{1}^{\prime} c_{N}-k_{1} x y^{N-1} \\
\frac{d y}{d t} & =k_{0} x-k_{0}^{\prime} y-(N-1) k_{1} x y^{N-1}-N k_{2} y^{N}+\left((N-1) k_{1}^{\prime}+N k_{2}^{\prime}\right) c_{N} \\
\frac{d c_{N}}{d t} & =k_{1} x y^{N-1}+k_{2} y^{N}-k_{1}^{\prime} c_{N}-k_{2}^{\prime} c_{N}
\end{aligned}
$$

The rate constants $a$ and $a^{\prime}$ control the rate of exchange between the bulk and the solution near the crystal surface (in effect, this represents a zero-dimensional model for spatial diffusion). The constants $k_{0}$ and $k_{0}^{\prime}$ control the rates of adsorption and desorption. The remaining rate constants pertain to the rates of nucleation and dissolution of clusters. Note that the probability to bring together $N$ molecules is taken to be proportional to the $\mathrm{N}$-th power of the concentration: this is equivalent to assuming the rate goes like $e^{-\beta N \Delta W}$ where $\Delta W$ is the work of formation per molecule of a cluster of size $N$ and using the lowdensity approximation that the free energy of a molecule in solution is proportional to the $\log$ of the concentration.

Notice that in the perspective of Eqs.(1) growth arises from the fact that the number of clusters $c_{N}$ ( $N$ given) increases until the whole surface is covered. Thus, the total solid mass is $M=N c_{N}$ and the growth velocity is $d M / d t=d N c_{N} / d t$. (In this zero-dimensional model, there is no distinction between growth within a layer and growth perpindicular to the surface.) In actual fact, in addition to this mechanism one expects that the formation of bigger clusters from smaller ones through, e.g., the process $C_{N}+Y \rightarrow C_{N+1}$ should 
also contribute to growth. In this Section, we will limit ourselves to the model of Eqs.(1), which will be used as a reference for sorting out the different growth regimes in a transparent manner and as a basis for comparison with the Monte Carlo simulations. This approximation is expected to be valid for high supersaturations where step growth is less important. Finally, we only consider the process in which one molecule is directly incorporated from the solution since, for the conditions considered here, we expect this to be dominant over processes involving two or more molecules coming from solution.

\section{A. A simplified model}

To illustrate the basic mechanism leading to a change in growth regimes, we first ignore depletion of the protein in solution near the interface so that $a=a^{\prime}=0$ and $x=x_{0}$ and neglect all dissolution processes so that $k_{0}^{\prime}=k_{1}^{\prime}=k_{2}^{\prime}=0$. This limit should describe to a good approximation crystals growing in the kinetically-limited regime. What remains is then the simplified dynamics,

$$
\begin{aligned}
\frac{d y}{d t} & =k_{0} x-(N-1) k_{1} x y^{N-1}-N k_{2} y^{N} \\
\frac{d c_{N}}{d t} & =k_{1} x y^{N-1}+k_{2} y^{N}
\end{aligned}
$$

Since there is no detailed balance, there is no equilibrium state. However, a steady state

where $\frac{d y}{d t}=0$ exists, implying a constant growth velocity, $N \frac{d c_{N}}{d t}=v$. The concentration of adsorbed molecules is then determined by

$$
0=k_{0} x-(N-1) k_{1} x y^{N-1}-N k_{2} y^{N}
$$

and the velocity is

$$
v=N k_{1} x y^{N-1}+N k_{2} y^{N}
$$

When the concentration of protein in solution is small compared to that adsorbed on the surface, $x<<y$, one finds

$$
\begin{aligned}
& y=\left(\frac{k_{0} x}{N k_{2}}\right)^{1 / N}+\ldots \\
& v=k_{0} x+\ldots
\end{aligned}
$$


while the reverse circumstance, $x>>y$, gives

$$
\begin{aligned}
& y=\left(\frac{k_{0}}{(N-1) k_{1}}\right)^{1 /(N-1)}+\ldots \\
& v=\frac{N}{N-1} k_{0} x+\ldots
\end{aligned}
$$

There is therefore a crossover from small $x$ behavior, where the aggregation process, $N y \rightleftarrows$ $c_{N}$, dominates with velocity $v=k_{0} x$ to the large $x$ behavior where the $x+(N-1) y \rightleftarrows c_{N}$

process dominates with velocity $v=\frac{N}{N-1} k_{0} x$. Since this transition occurs in the normal growth regime - below the usual roughening transition - the growth rates are, in these limits, linear functions of the concentration, as expected. Small and medium sized clusters are those contributing the most in the switching, since for large $N$ the growth velocities of the two regimes become practically indistinguishable. As we will discuss elsewhere, to get a more detailed sense of the interplay between these mechanisms, a simple model can be formulated for which numerically-exact results can be obtained using the theory of finite Markov processes [32].

\section{B. Full model}

In the simplified model, all dissolution processes were neglected. Experimental investigations [33, 34] have shown, however, that this is not valid for protein crystals growing at low driving forces. Here, we therefore incorporate desorption and dissolution processes in the full model. Including the desorption of surface molecules, $k_{0}^{\prime} \neq 0$, does not change the picture dramatically: the small $\mathrm{x}$ behavior is no longer linear but there is still a crossover between one well-defined growth mode at small $x$ to another, faster mode at large $x$ as in the simple model. Including all of the evaporation processes, $k_{1,2}^{\prime} \neq 0$, however, does change the picture significantly. First, detailed balance defines an equilibrium state at some finite value of $x_{0}$. For bulk concentrations above this value, growth occurs but it will eventually end when a steady (equilibrium) state is reached. In between, a quasi-steady growth regime occurs during which a growth velocity can be defined. Detailed numerical solutions of the model again show a crossover in the growth velocity as the bulk concentration increases. The requirement of detailed balance at equilibrium, i.e. that the forward rates and backward 
rates of all processes are equal, gives

$$
\begin{aligned}
a x_{0} & =a^{\prime} x^{*} \\
k_{0} x^{*} & =k_{0}^{\prime} y^{*} \\
k_{1}^{\prime} c_{N}^{*} & =k_{1} x^{*} y^{* N-1} \\
k_{2} y^{* N} & =k_{2}^{\prime} c_{N}^{*}
\end{aligned}
$$

where $x^{*}$ is the equilibrium value of $x$, etc. Given a value for the concentration in the bulk solution, these relations determine the equilibrium values of all of the other quantities as well as imposing the consistency relation

$$
k_{0}^{\prime} k_{1} k_{2}^{\prime}=k_{0} k_{1}^{\prime} k_{2} .
$$

Supersaturation at the surface is defined as $\log \left(x / x^{*}\right)$. To illustrate the general behavior of this model, we have solved for the time-dependence of the various quantities for the case that $a=a^{\prime}=0.04 k_{0}, k_{1}=0.02 k_{0}, k_{1}^{\prime}=k_{2}^{\prime}=0.004 k_{0}, k_{0}^{\prime}=0.5 k_{0}, k_{2}=0.01 k_{0}, N=2$ with initial conditions $x_{0}(0)=1$, and $x(0)=y(0)=c(0)=0$. Here the original time $t$ is normalized to $k_{0} t$. The value of $k_{0}^{\prime}$ reflects the idea that once adsorbed, a molecule spends on average a long time on the surface before being desorbed back to the bulk. The choice $k_{2}<k_{1}$ amounts to assuming that the activation barrier for aggregation alone is larger than that for adsorption and aggregation. Aside from these assumptions and the physical requirements that the rates at which molecules join a cluster, $k_{1}$ and $k_{2}$, are greater than the rates for the reverse processes and the detailed balance condition, Eq, 8 , these values are somewhat flexible. Given an initially flat surface, $c_{N}(0)=0$, islands will form causing the mass of the surface to increase until, at long times, equilibrium is reached. At intermediate times, a more-or-less steady growth regime is reached, see Fig. 1, corresponding to a plateau in the growth velocity, $\frac{d c_{N}}{d t}$, which defines a quasi-steady state growth velocity as shown in Fig. 2 , Taking the maximum growth velocity as a measure of the typical velocity at intermediate times, one again finds a crossover between small $x$ and large $x$ regimes as illustrated in Figure 3 . 


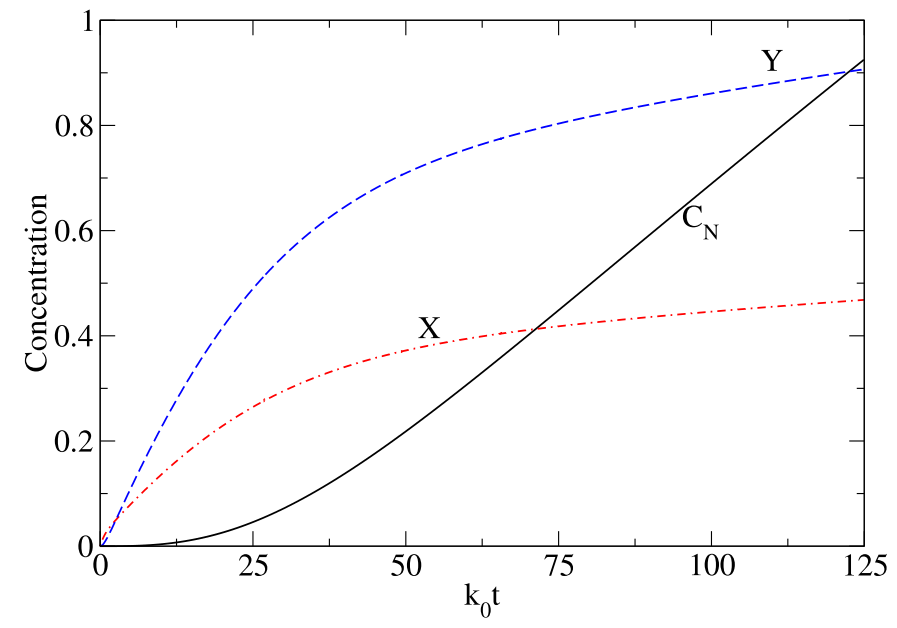

FIG. 1: Time-dependence of the quantities $x(t), y(t)$ and $c_{N}(t)$ as results from a numerical solution of the full model, Eqs.(11), with the parameters given in the text.

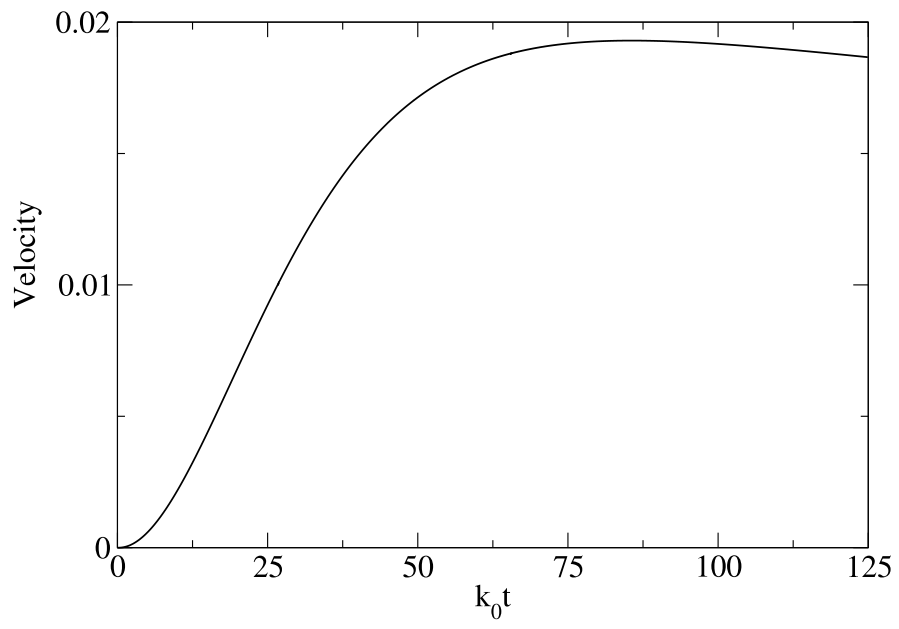

FIG. 2: The dimensionless growth velocity, $\frac{d N c_{N}}{d k_{0} t}$, as a function of time for the same parameters as used in Fig. 1. The supersaturation in this case is $\log \left(x / x^{*}\right) \approx-0.84$.

\section{ONE-DIMENSIONAL KINETIC MONTE CARLO MODEL}

In order to study both the possibility of multiple growth regimes without vanishing nucleation barrier and the roughening transition, we have performed Kinetic Monte Carlo simulations of the growth of a simple, one-dimensional surface in the spirit of the Solid-On-Solid 


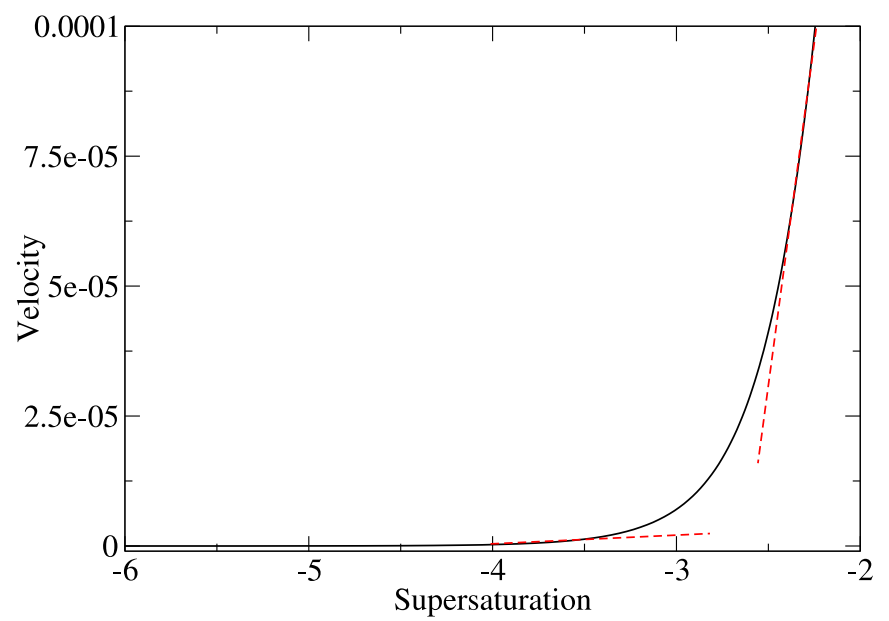

FIG. 3: Maximal growth velocity as a function of supersaturation, $\log \left(x / x^{*}\right)$, for the parameters given in the text. The broken lines extrapolate the values into asymptotic regions.

(SOS) model[35]. The system consists of a set of growth sites arranged as a one-dimensional lattice. There are $N$ sites, each characterized by a height $h_{i}$ where $i=0, \ldots, N-1$. Periodic boundaries are used so that formally, one has $h_{N}=h_{0}$. The idea is that molecules randomly land on the surface at some specified rate $\nu_{\text {hit }}$ representing the diffusion of molecules onto the surface. Once on the surface, a molecule attempts to leave the surface at a rate $\nu_{\text {evap }}$. Physically, $\nu_{h i t}$ will be determined by the concentration of the molecules in solution and the rate at which they move while $\nu_{\text {evap }}$ is a measure of how fast a molecule makes an attempt to leave the surface.

Nucleation is anomalous in one dimension due to the fact that the "surface area" of a cluster does not depend on its size. We therefore introduce a rule designed to capture the most important feature of surface nucleation which is the existence of a critical cluster size. Specifically, molecules are not allowed to leave the surface if they are part of a cluster that is of a specified critical size or larger. The critical size $n_{c}$ is a parameter. Molecule $j$ is "part of a cluster" of size $n$ if there are $n$ contiguous sites, including site $j$, with heights the same as or greater than $h_{j}$. Thus, small clusters can form spontaneously and dissociate but a cluster at or above the critical size is stable.

A time step $d t=1 /\left(\nu_{\text {hit }}+\nu_{\text {evap }}\right)$ is defined and the dynamics of the kinetic Monte Carlo algorithm are as follows: 
1. At time $t$ the system has some configuration of heights and total mass $M(t)=\sum_{i=0}^{N} h_{i}$ and roughness $R(t)=\frac{1}{N} \sum_{i=0}^{N-1}\left|h_{i}-h_{i-1}\right|$. Roughness could alternatively be characterized by the variance of the heights, the correlation length, ...

2. The following is performed $N$ times:

(a) Choose a random site, $j$.

(b) choose a random number $u \in[0,1]$ and set $h_{j} \rightarrow h_{j}+1$ if $u<\nu_{\text {hit }} d t$.

(c) if the height is not increased, (this happens with probability $1-\nu_{\text {hit }} d t=\nu_{\text {evap }} d t$ ), then remove a molecule, $h_{j} \rightarrow h_{j}-1$ if site $j$ is not part of a supercritical cluster.

3. Set $t \rightarrow t+d t$.

4. Return to step 1 until the desired number of cycles is completed.

As it stands, there are only two meaningful parameters: the ratio of the two rates, $\nu_{\text {hit }} / \nu_{\text {evap }}$ and the size of the critical cluster, $n_{c}$. Since super-critical clusters are absolutely stable there is no equilibrium state: clusters form no matter what value the parameters are given, as in the simplified form of the mean-field model discussed above. The growth rate is calculated as $(M(t+d t)-M(t)) / d t$ and is normalized to the number of sites to give the corresponding growth velocity. In the simulations, we also allow for the presence of "defects", localized regions in which the critical cluster size is smaller than elsewhere, to serve as sites for heterogeneous nucleation.

Simulations have been performed under three circumstances: no defects, a "wall" defect and a "spiral" defect. The "wall" refers to a set of sites, say sites $0, \ldots, n_{c}$ for which the evaporation rate is zero. These therefore grow very fast and serve as a source for heterogeneous nucleation. For the "spiral" defect, the critical nucleus for site 0 is set to one, for site 1 it is set to 1 , and so forth up to site $n_{c}$. This is not meant to realistically model a spiral defect, but rather to test whether the results are sensitive to the shape and nature of the defect. The name indicates the similarity in shape of the resulting defect to a projection of a spiral defect onto two dimensions. In fact, we observe no qualitative difference in the results using the two different defects. The main effect is that at low supersaturation, $\nu_{\text {hit }} / \nu_{\text {evap }}<<1$, the rate of growth in the system with no defects is dominated by the time taken for nucleation to occur - by comparison, step growth happens relatively quickly. Thus, the measured 

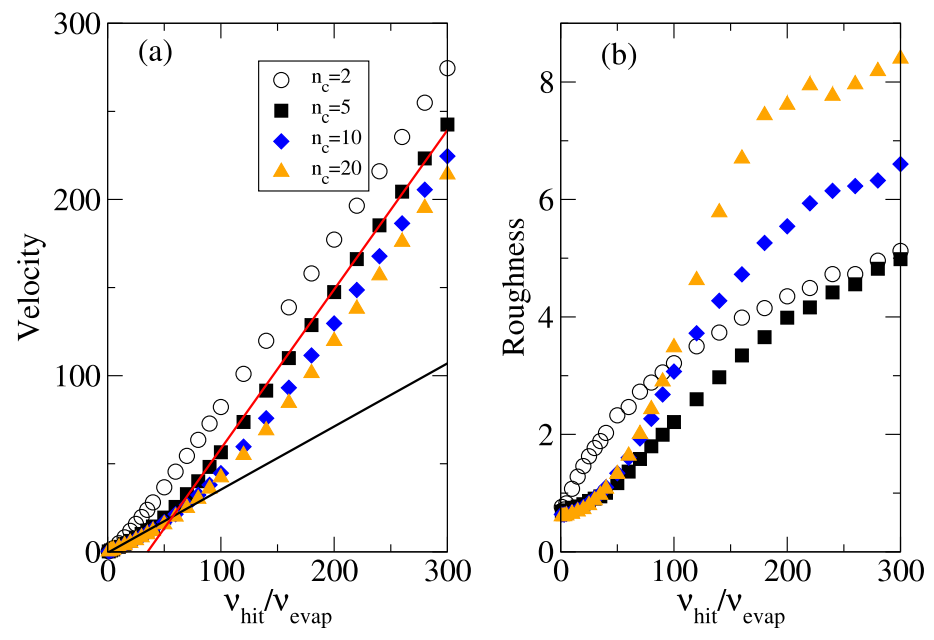

FIG. 4: Growth velocity $\frac{d M}{d \nu_{\text {evap }} t}$ (panel a) and roughness (panel b) as functions of the hit rate for various choices of the critical size, $n_{c}$ in the presence of a spiral defect.

rate of increase of the solid is basically a measure of the nucleation rate and is not directly relevant to the rate of step growth. However, the same qualitative effects are seen with and without defects. Intuitively, one expects that if $\nu_{\text {hit }}<<\nu_{\text {evap }}$ then nucleation will be rare and growth will be dominated by attachment to existing super-critical clusters, i.e. smooth step growth. When the hit rate increases, nucleation becomes more probable until at some point nucleation can occur easily and growth will be a combination of growth of existing clusters and nucleation of new clusters. Simulations were performed for a system consisting of 1500 sites and the velocity, defined as the rate of change of the total mass, was averaged over 1000 cycles.

Figure 4 shows the rate of growth of the surface as a function of the hit rate for various choices of the size of the critical cluster when a spiral defect is present. For a small critical cluster, $n_{c}=2$, nucleation is almost instantaneous for all but the lowest values of $\nu_{\text {hit }} / \nu_{\text {evap }}$ : growth is dominated by nucleation and the growth velocity increases more or less linearly with the hit rate. For larger critical sizes, two different growth regimes can be distinguished in the Figure. At small hit rates, growth is dominated by heterogeneous nucleation at the defect with the subsequent growth of steps away from the defect. This growth by aggregation is analogous to the $N Y \rightarrow C_{N}$ scenario in the mean-field model. However, at high hit rates, nucleation becomes important and the growth rate increases due to the 


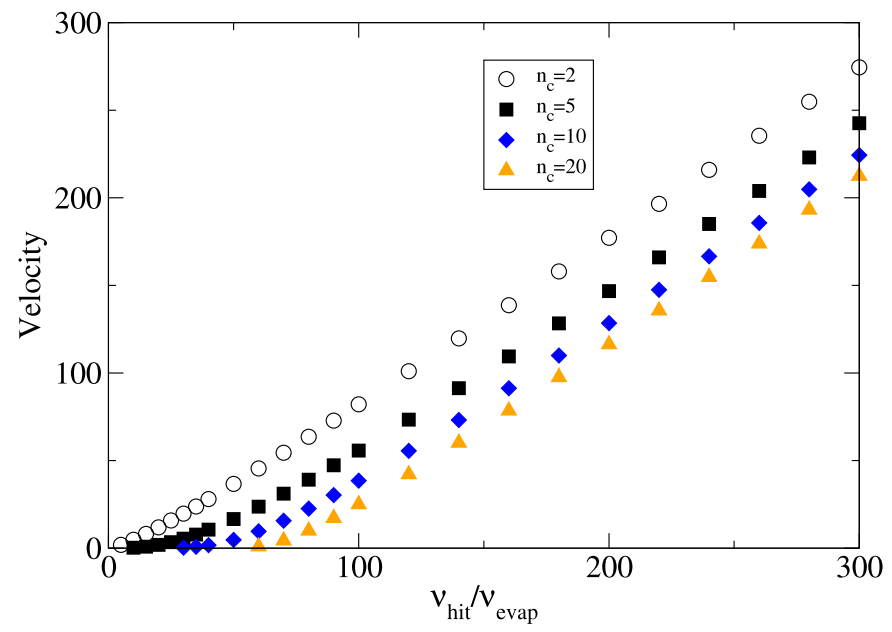

FIG. 5: Growth velocity as a function of hit rate for various choices of the critical size, $n_{c}$ with no defect. The initial growth rate is very small and is dominated by the nucleation rate.

combination of processes with nucleation dominating at high hit rates. This is analogous to the $(N-1) Y+X \rightarrow C_{N}$ process in the mean-field model. The point of crossover between the regimes increases with increasing critical size and corresponds to a roughening transition as is illustrated in Fig. 4b. Note that for the smallest critical cluster size, the transition region is too small to see in this figure. For critical clusters larger than 2 and for small supersaturations, the roughness is virtually independent of the critical cluster size and increases slowly with the supersaturation while at higher supersaturation, the transition to the rapid growth regime is also signaled by a dramatic increase in roughness.

Finally, Fig. 5 shows the growth rate when no defect is present. The main difference from the previous results is that at low supersaturations, the growth rate is very small since growth only occurs after the nucleation of a super-critical cluster. Once such a cluster forms, the growth of a new layer of crystal is quite rapid so that the overall growth rate is dominated by the nucleation rate. Nevertheless, a clear crossover from this slow growth regime, where most mass is added by step growth, to a fast regime, where nucleation plays an important role in adding mass, is evident. 


\section{CONCLUSIONS}

Within the context of classical nucleation theory, kinetic roughening is understood as occurring when the supersaturation is so high that the size of the critical cluster becomes smaller than one molecule[1]. At low supersaturations, growth is dominated by the heterogeneous nucleation of steps that then grow smoothly to cover the crystal surface while at high supersaturation, above the transition, homogeneous nucleation also occurs leading to rapid growth of a rough surface. In this paper, the possibility of a similar transition for finite-sized critical clusters has been investigated. Two models for the growth of crystal surfaces which show a transition from a slow to a fast growth regime were presented. In the mean field model, adsorption onto the surface with subsequent surface diffusion and aggregation competes with direct formation of clusters to give two different growth regimes for the formation of islands of new crystal. At low bulk concentrations, the adsorption and aggregation mechanism are dominant whereas at high bulk concentrations, the formation of critical clusters by the formation of subcritical aggregates with direct incorporation of material from solution dominates. The difference in growth rates is greatest when the critical cluster is small (being a factor of 2 for a critical size of 2). These results elaborate on previous theoretical work that indicated the importance of direct incorporation of material from the bulk[31].

A one-dimensional Kinetic Monte Carlo model based on the well-known SOS model showed both different growth regimes and roughening. The main difference from the usual SOS model is the definition of a critical cluster size to simulate nucleation as it actually occurs on a two-dimensional surface. This model showed a clear cross-over between a smooth, step-growth dominated regime at low supersaturation and a rough, nucleation-dominated regime at high supersaturation thus exhibiting the observed features of the kinetic roughening transition. The growth velocities both below and above the transition are roughly linear functions of the hit rate (Fig. 4), or in physical terms, the concentration, just as in the mean-field model (see, e.g., Eqs.(15, 6) ).

The main conclusion from this work is that the kinetic roughening-like transitions do not necessarily require the vanishing of the critical cluster size. Instead, "kinetic" effects suffice to give qualitatively different growth regimes and roughening of the surface. In future work, it is intended to further elaborate the simple mean field model presented here to give a 
semi-quantitative description of the results of simulation as well as of experiment. Finally, it is noteworthy that the experiments revealing the kinetic roughening mechanisms that

motivated the present study[16] were done using gel to quench convection which brings one close to microgravity conditions. It might be expected that a comparison under real microgravity conditions would reveal further important features.

\section{Acknowledgments}

This work was supported by the European Space Agency under contract number ESA AO-2004-070.

[1] Y. Saito, Statistical Physics of Crystal Growth (World Scientific, Singapore, 1996).

[2] A. J. Malkin, T. A. Land, Y. G. Kuznetsov, A. McPherson, and J. J. DeYoreo, Phys. Rev. Lett. 75, 2778 (1995).

[3] T. A. Land, A. J. Malkin, Y. G. Kuznetsov, A. McPherson, and J. J. De Yoreo, Phys. Rev. Lett. 75, 2774 (1995).

[4] A. J. Malkin, Y. G. Kuznetsov, T. A. Land, and J. J. De Yoreo, Nat. Struct. Biol. 2, 956 (1995).

[5] S. T. Yau and P. G. Vekilov, Nature 406, 494 (2000).

[6] I. Reviakine, J. Am. Chem. Soc. 125, 11684 (2003).

[7] N. K. Burton, N. Cabrera, and F. C. Frank, Phil. Trans. R. Soc. Lond. A 243, 299 (1951).

[8] K. A. Jackson, Growth and Perfection of Crystals (Chapmann and Hill, London, 1958).

[9] A. E. Nielsen, in International Series of Monographs on Analytical Chemistry, edited by R. Belcher and L. Gordon (Pergamon, New York, 1964), vol. 1.

[10] J. D. Weeks, Ordering in Strongly Fluctuating Condensed Matter Systems (Plenum Press, New York, 1986).

[11] M. Elwenspoek, J. Phys. A: Math. Gen. 20, 669 (1987).

[12] A. J. Malkin, A. A. Chernov, and I. V. Alexeev, J. Cryst. Growth 97, 765 (1989).

[13] I. N. Stranski and R. I. Kaischew, Z. Phys. Chem. B 26, 100 (1934).

[14] R. Kaischew and I. N. Z. Stranski, Phys. Chem. B 1937, 34, 427-432 34, 427 (1937). 
[15] S. Gorti, E. L. Forsythe, and M. L. Pusey, Cryst. Growth Des. 4, 691 (2004).

[16] M. Sleutel, R. Willaert, C. Evard, L. Wyns, and D. Maes, Crys. Growth and Design 9, 497 (2009).

[17] H. E. Buckley, Crystal Growth (John Wiley and Sons, New York, 1951).

[18] T. Hwa, M. Kardar, and M. Paczuski, 66, 441 (1990).

[19] M. Elbaum, 67, 2982 (1991).

[20] B. Mutaftschiev, in Handbook of Crystal Growth, edited by D. T. J. Hurle (North-Holland, 1993), vol. 1a.

[21] X.-Y. Liu, P. van Hoof, and P. Bennema, 71, 109 (1993).

[22] K. Izumi, Gan Ping;, M. Hashimoto, A. Toda, H. Miyaji, Y. Miyamoto, and Y. Nakagawa, Advances in the Understanding of Crystal Growth Mechanisms (North-Holland: Amsterdam, 1997).

[23] R. A. Laudise and A. A. Ballman, J. Phys. Chem. 64, 688 (1960).

[24] E. D. Kolb and R. A. Laudise, 49, 302 (1966).

[25] L. A. M. J. Jetten, H. J. Human, P. Bennema, and J. P. van der Eerden, 68, 503 (1984).

[26] X.-Y. Liu and P. Bennema, J. Cryst. Growth 139, 179 (1994).

[27] S. D. Durbin and G. Feher, J. Cryst. Growth 76, 583 (1986).

[28] S. Gorti, E. L. Forsythe, and M. L. Pusey, Cryst. Growth Des. 5, 473 (2005).

[29] A. Myers-Beaghton and D. Vvedensky, Phys. Rev. A 44, 2457 (1991).

[30] S. Harris and P. S̆milauer, Phys. Rev. B 50, 7952 (1994).

[31] R. A. Garza-Lopez, P. Bouchard, G. Nicolis, M. Sleutel, J. Brzezinski, and J. J. Kozak, J. Chem. Phys. 128, 114701 (2008).

[32] J. J. Kozak, G. Nicolis, J. F. Lutsko, and V. Basios (2009), in preparation.

[33] K. Chen and P. G. Vekilov, Phys. Rev. E 66, 21606 (2002).

[34] M. Sleutel, C. Vanhee, C. V. de Weerdt, K. Deconniere, D. Maes, L. Wyns, and R. Willaert, Cryst. Growth and Design 8, 1173 (2008).

[35] G. H. Gilmer and P. Bennema, J. Appl. Phys. 43, 1347 (1972). 